\title{
Análisis crítico de los recursos educativos digitales propuestos por el Ministerio de Educación de Chile en el texto escolar del estudiante de las asignaturas de Biología y Química de Educación Secundaria
}

\author{
Yorka Tatiana Ortiz ${ }^{1}$ - Cecilia Alejandra Pino Villalobos ${ }^{2}$
}

Recepción: 10-10-2020 / Aceptación: 12-01-2021

\section{Resumen}

Los recursos digitales son una herramienta importante en la vida cotidiana de toda la sociedad, por lo cual es necesario conocer la calidad de estos recursos, en especial en el ámbito educativo, como los propuestos por el Ministerio de Educación en los textos escolares.

Esta investigación, de tipo cualitativa, da cuenta de los recursos educativos digitales propuestos en los textos escolares de Biología y Química de Educación Secundaria chilena del año 2018. Para ello se buscó conocer la disponibilidad; rol pedagógico y calidad de dichos recursos, a través de análisis de contenido en una muestra de tres textos escolares de Biología: 1, 2 y 3-4 de secundaria y tres de Química: 1, 2 y 3-4, también de secundaria.

Se evidencia que existen 24 recursos no disponibles en los textos de Biología, de un total de 76; y 67 recursos de 148 en los textos de Química. En el rol pedagógico de los recursos predomina el informativo en desmedro del demostrativo e interactivo en ambas disciplinas. Finalmente, en cuanto a la calidad de los recursos definida como excelente, regular y deficiente, se observa que los textos de Química proporcionan recursos de mejor calidad.

Palabras clave: Recursos digitales, Biología, Química, Educación, textos escolares.

\begin{abstract}
Digital resources are an important tool in the daily life of the entire society, so it is necessary to know the quality of these resources, especially in the educational field, such as those proposed by the Ministry of Education in school textbooks.

This qualitative research provides an account of the digital educational resources proposed in the Chilean Secondary Education Biology and Chemistry textbooks for the year 2018. For this, we sought to know the availability, pedagogical role and quality of these resources, through content analysis in a sample of three school texts on Biology: 1,2 and 3-4 from secondary school and three from Chemistry: 1,2 and 3-4, also from secondary school.

It is evident that there are 24 resources not available in Biology texts, out of a total of 76 ; and 67 resources out of 148 in Chemistry texts. In the pedagogical role of resources, the informative predominates to the detriment of the demonstrative and interactive in both disciplines. Finally, regarding the quality of the resources defined as excellent, regular and poor, it is observed that the Chemistry texts provide better quality resources.
\end{abstract}

Key words: Digital resources, Biology, Chemistry, Education, textbooks.

1. Doctora en Investigación Transdisciplinar en Educación con mención Internacional de especialidad Informática Educativa; Académica; email: yortiz@ulagos.cl; ORCID: https://orcid.org/0000-0002-2870-2253

2. Maestra en Ciencias de la Educación: Pedagogía en Biología y Ciencias Naturales; Académica; email: cpino@ulagos.cl; ORCID: https://orcid.org/0000-0001-6461-8553 


\section{Introducción}

Nos situamos en una sociedad que se caracteriza por un constante cambio, a la cual algunos autores como Castells (2001) denominan Sociedad de la Información o del Conocimiento; que repercute en el ámbito educativo, ya que se apropia de la tecnología educativa de manera transversal a todas las disciplinas del sistema escolar. Según señala Ortiz (2016), es importante que en el proceso educativo no se reemplacen los recursos educativos tradicionales por las tecnologías educativas, sino que se debe propiciar un cambio metodológico y didáctico en las prácticas docentes (Moreira, 2011).

Waldegg (2002) señala que una de las principales ventajas de la utilización de las tecnologías apunta en recapturar el mundo real, y reabrirlo al estudiante en el interior del aula, con amplias posibilidades de interacción y manipulación de su parte. Esto significa, según la autora, emular la actividad científica aprovechando el hecho de que las tecnologías; logran representaciones ejecutables que permiten al estudiante modificar condiciones, controlar variables y manipular el fenómeno estudiado.

Autores como Waldegg (2002) y Capuano (2011) propugnan por la integración de las tecnologías de la información y la comunicación (TIC) ${ }^{3}$ para el aprendizaje de las ciencias, afirmando que, utilizadas adecuadamente, tienen la capacidad de:
- Presentar los materiales a través de múltiples medios y canales.

- Motivar e involucrar a los estudiantes en actividades de aprendizaje significativas.

- Proporcionar representaciones gráficas de conceptos y modelos abstractos.

- Mejorar el pensamiento crítico y otras habilidades, y procesos cognitivos superiores.

- Posibilitar el uso de la información adquirida para resolver problemas y para explicar los fenómenos del entorno.

- Permitir el acceso a la investigación científica y el contacto con científicos y bases de datos reales.

- Ofrecer a maestros y estudiantes una plataforma a través de la cual pueden comunicarse con sus pares de lugares distantes; intercambiar trabajo, desarrollar investigaciones y funcionar como si no hubiera fronteras geográficas.

El impacto de las tecnologías en el proceso educativo es algo innegable. Capuano (2011) señala que son recursos innovadores, que permiten diseñar un conjunto de estrategias en las prácticas docentes, capaces de producir una verdadera revolución educativa en general y en la enseñanza de las ciencias naturales en particular. En específico, señala que la presencia de las TIC en la enseñanza de las ciencias naturales es variada y se da en todos los ámbitos: en las clases teóricas, general-

3. Dentro del documento, las autoras se referirán a este término mediante este acrónimo. 
mente expositivas en las que se presentan determinados conceptos; en clases de resolución de problemas y en clases de realización de experimentos.

Ramírez (2011) señala que la utilización de las TIC en el aprendizaje de las ciencias es para comunicarse, recabar información, retroalimentación, simular y visualizar situaciones; para la obtención y el tratamiento de datos, etc. Se trata de un recurso útil que contribuye a mostrar una visión actualizada de la actividad científica.

\section{Textos Escolares y TIC}

Según Naranjo y Candela (2010), los libros de texto son importantes en el proceso de enseñanza debido a su aporte en el proceso de instrucción. Los profesores encuentran en estos textos sugerencias metodológicas, ejercicios y actividades; ideas para motivar a los estudiantes en sus clases (García, 1996). Las actividades presentes en los textos escolares deben ser variadas, presentar diferentes estrategias, habilidades y competencias que planteen desafíos constantes para los estudiantes (Pino y Díaz-Levicoy 2013). También, los textos escolares deben presentar coherencia con las directrices curriculares del sistema educacional (Pino, Diaz-Levicoy y Piñeiro 2014).

El Ministerio de Educación chileno (2008) menciona que el texto escolar es un medio para que los educandos, de sectores socioeconómicamente vulnerables, puedan acceder a una educación de calidad, equidad y enriquecimiento cultural. Según esto, es necesario que se consideren las tecnologías en los textos escolares, de acuerdo a lo declarado en las Bases Curriculares (MINEDUC, 2019). Este documento expresa que la utilización de la tecnología como herramienta de trabajo, implica dominar las posibilidades que ofrece como, asimismo, darle un uso creativo e innovador.

García-Valcárcel y Hernández (2013) señalan que los libros de texto deben garantizar cuatro aspectos fundamentales:

1. El valor de la información (calidad científica).

2. Adaptación de la información al contenido ambiental y cultural.

3. Adaptación de los contenidos y propuestas metodológicas al estado madurativo de los alumnos.

4. Coherencia pedagógica y fundamentación en teorías del aprendizaje bien sustentadas.

Los antecedentes anteriores motivan el desarrollo de esta investigación, donde se analizan los recursos tecnológicos propuestos por el Ministerio en los textos escolares de Educación Secundaria en las asignaturas de Biología y Química para conocer su disponibilidad, rol pedagógico y calidad.

\section{Metodología}

Esta investigación se realizó el año 2019, analizando textos utilizados en el año 2018; y se enmarca en el paradigma cualitativo. El método utilizado es el estudio de contenido (López, 2002), caracterizado por seguir un proceso cíclico e inductivo. Los libros de texto 
utilizados como muestra fueron elegidos por muestreo intencional de selección controlada (Hernández, Fernández y Baptista, 2010).

Se analizaron los textos escolares de $1^{\circ}$ a $4^{\circ}$ año de Educación Secundaria de Biología y Química (ver figura 1), que son elaborados y editados de acuerdo a las directrices curriculares del Ministerio de Educación chileno.

El proceso investigativo se dividió en cuatro etapas:

a. Registro de recursos TIC: se utilizó una matriz construida en Excel, donde se registraron los recursos presentes en los textos escolares de las asignaturas de Biología y Química de primero a cuarto año de enseñanza Secundaria.

b. Registro de la disponibilidad: se ingresó a cada uno de los recursos para determinar la vigencia.

\section{c. Clasificación de acuerdo a su rol pedagógico:}

Meneses (2007) señala que los medios didácticos asumen diferentes orientaciones, desde los que facilitan información a otros que ofrecen interacciones facilitadoras de aprendizajes a los alumnos. Siendo así, y al considerar la función que el recurso va a cumplir en el acto educativo, se definen tres roles de acuerdo a las características técnicas y pedagógicas de estos:

- Informativo, se caracteriza por un rol pasivo de entrega de información, es decir, cuando un recurso entrega información usando distintos medios, con el objetivo de complementar la información que entregan los textos.

- Demostrativo, donde el estudiante pasa de ser pasivo consumidor de información a activos aprendices que usan esa información, la procesan y sirven de base en la toma de decisiones. Es decir, avanzan en cuanto a que no solo presentan la información, sino que realizan exposiciones específicas de temáticas relacionadas con la asignatura tratada.

- Interactivo, requieren de los estudiantes una alta capacidad de seguir aprendiendo; es un medio dinámico y participativo, que promueve un estilo único de

\begin{tabular}{|l|l|}
\hline \multirow{4}{*}{ Textos Biología } & López y Pereda (2015). Biología $1^{\circ}$ Medio \\
\cline { 2 - 2 } & Chaucon y Vargas (2015). Biología $2^{\circ}$ Medio \\
\cline { 2 - 2 } & $\begin{array}{l}\text { Abarca, Flores, Caro, Lepe, Fernández, Pepper y Poblete (2014). } 3^{\circ} \\
\text { y } 4^{\circ} \text { Medio }\end{array}$ \\
\hline \multirow{3}{*}{ Textos Química } & Estrada, Gómez y Lara (2015). Química $1^{\circ}$ Medio \\
\cline { 2 - 2 } & Cabello (2015). Química $2^{\circ}$ Medio \\
\cline { 2 - 2 } & Cabello (2015b). Química $3^{\circ}$ y $4^{\circ}$ Medio \\
\hline
\end{tabular}

Figura 1. Listado de textos escolares analizados. 
aprendizaje; que da énfasis a los recursos, las relaciones y la exploración ${ }^{4}$.

- Evaluación de la calidad del recurso: Los recursos digitales fueron evaluados por una pauta, adaptación de la "Escala de evaluación de recurso educativo propuesta por una educación para una Aldea Global 2006" del Instituto de Informática Educativa de la Universidad de La Frontera, Chile. Este instrumento fue validado por expertos del área educación, informática educativa e informática (ver figura 2).

\section{Resultados}

Los resultados se presentan de acuerdo a las etapas de investigación, iniciando en el registro de disponibilidad; luego de acuerdo al rol pedagógico $\mathrm{y}$, finalmente, fueron evaluados considerando la calidad.

\section{a. Registro de disponibilidad}

El primer análisis realizado es la disponibilidad de los recursos tecnológicos, (ver tablas 1 y 2$)$.

Es importante destacar que, de los 260 recursos educativos indicados en los textos escolares, existen 91 recursos no disponibles. Esta información es relevante porque deja en evidencia la desactualización en que se encuentran los textos del estudiante.

\section{Rol pedagógico}

Considerando la función que el recurso va a cumplir en el acto educativo, se definen tres roles de acuerdo a características técnicas y pedagógicas: informativo, demostrativo e interactivo (ver tabla 3 y 4 ).

\begin{tabular}{|l|l|}
\hline \multirow{4}{*}{ Dimensiones Pauta de evaluación } & Velocidad \\
\cline { 2 - 2 } & $\begin{array}{l}\text { Primera impresión - apariencia } \\
\text { general }\end{array}$ \\
\cline { 2 - 2 } & Facilidad para la navegación \\
\cline { 2 - 2 } & $\begin{array}{l}\text { Uso de gráficos / imágenes / sonidos / } \\
\text { videos }\end{array}$ \\
\cline { 2 - 2 } & Contenido / Información \\
\cline { 2 - 2 } & Actualización \\
\cline { 2 - 2 } & Disponibilidad de información \\
\hline
\end{tabular}

Figura 2. Dimensiones Pauta de evaluación.

4. Extraído de: Una Educación para una Aldea Global, Instituto de Informática Educativa. Universidad de La Frontera. 2006 
Tabla 1. Disponibilidad de recursos en textos de Biología

\begin{tabular}{|l|c|c|c|c|}
\cline { 2 - 5 } \multicolumn{1}{c|}{} & $\mathbf{1}^{\circ} \mathbf{M e d i o}$ & $\mathbf{2}^{\circ}$ Medio & $\mathbf{3}^{\circ} \mathbf{y} \mathbf{4}^{\circ}$ Medio & Total \\
\hline Disponibles & 8 & 14 & 30 & 52 \\
\hline No disponibles & 11 & 8 & 5 & 24 \\
\hline Total & 19 & 22 & 35 & 76 \\
\hline
\end{tabular}

Tabla 2. Disponibilidad de recursos en textos de Química

\begin{tabular}{|l|c|c|c|c|}
\cline { 2 - 5 } \multicolumn{1}{c|}{} & $\mathbf{1}^{\circ}$ Medio & $\mathbf{2}^{\circ}$ Medio & $\mathbf{3}^{\circ} \mathbf{y} \mathbf{4}^{\circ}$ Medio & Total \\
\hline Disponibles & 33 & 43 & 41 & 117 \\
\hline No disponibles & 19 & 21 & 27 & 67 \\
\hline Total & 52 & 64 & 68 & 148 \\
\hline
\end{tabular}

Tabla 3. Rol pedagógico de los recursos de Biología

\begin{tabular}{|l|c|c|c|}
\cline { 2 - 4 } \multicolumn{1}{c|}{} & Primer año & Segundo año & Tercer y Cuarto año \\
\hline Informativo & 5 & 13 & 23 \\
\hline Demostrativo & 2 & 1 & 6 \\
\hline Interactivo & 1 & 0 & 1 \\
\hline Total & 8 & 14 & 30 \\
\hline
\end{tabular}

Tabla 4. Rol pedagógico de los recursos de Química

\begin{tabular}{|c|c|c|c|}
\cline { 2 - 4 } \multicolumn{1}{c|}{} & Primer año & Segundo año & Tercer y cuarto año \\
\hline Informativo & 28 & 34 & 36 \\
\hline Demostrativo & 4 & 5 & 4 \\
\hline Interactivo & 1 & 4 & 1 \\
\hline Total & 33 & 43 & 41 \\
\hline
\end{tabular}


$\mathrm{Al}$ analizar los resultados expuestos en las tablas 3 y 4 se evidencia cómo decrece la cantidad de recursos, desde los informativos a los interactivos; en ambas ciencias, siendo el grueso de los recursos de tipo informativo como se representa en las figuras 3 y 4 .

\section{Evaluación de la calidad del recurso}

La calidad de los recursos educativos, al aplicar la pauta creada, entrega los siguientes re- sultados (ver tabla 5 y 6); y deja de manifiesto una distribución heterogénea, destacando en la asignatura de Química más recursos evaluados como excelentes que en Biología, desde segundo a cuarto año de secundaria. Pese a esto, si se concentra la atención en los considerados regulares y deficientes, suman $63.4 \%$ para los recursos de Biología y 44.8\% para los recursos de Química; lo que representa un gran número de recursos que no serían de la calidad esperable para el proceso educativo.

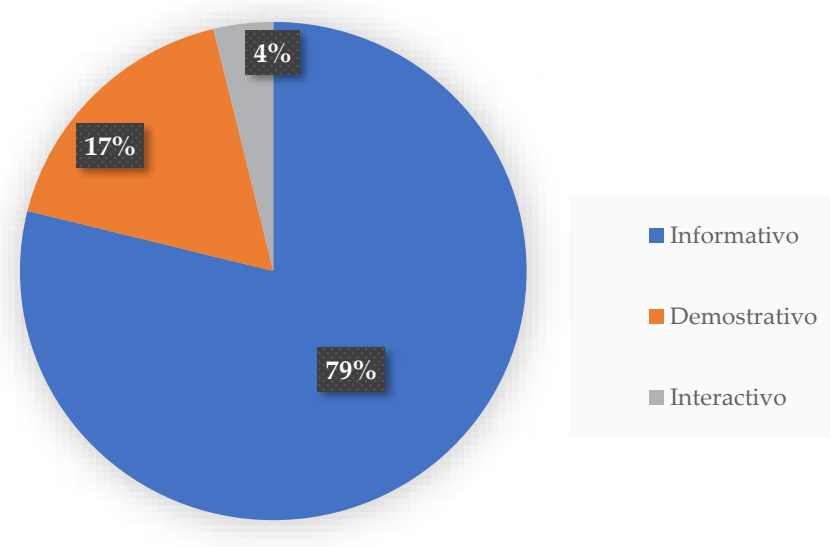

Figura 3. Rol pedagógico de los recursos TIC en las series de textos escolares de Bilogía.

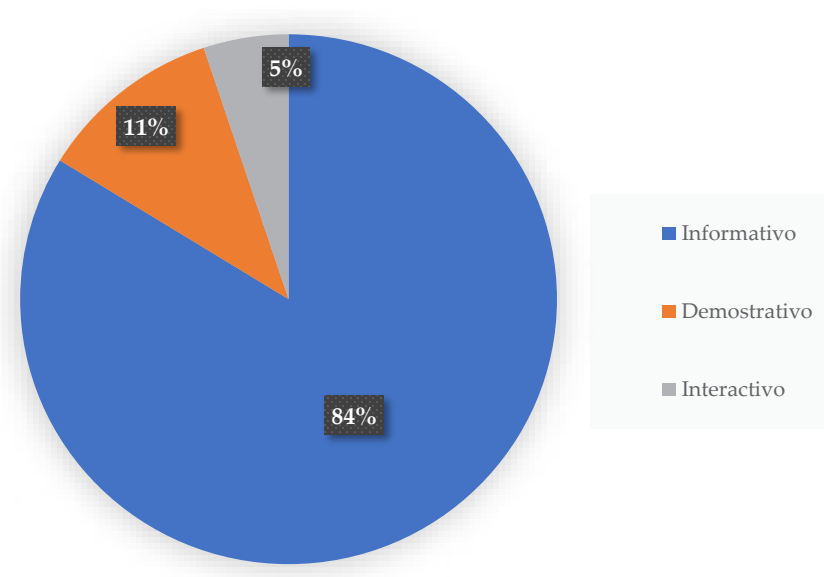

Figura 4. Rol pedagógico de los recursos TIC en las series de textos escolares de Química. 
Tabla 5. Evaluación de los recursos de Biología

\begin{tabular}{|l|c|c|c|}
\cline { 2 - 4 } \multicolumn{1}{c|}{} & Primer año & Segundo año & Tercer y Cuarto año \\
\hline Excelente & 1 & 4 & 14 \\
\hline Regular & 3 & 9 & 10 \\
\hline Deficiente & 4 & 1 & 6 \\
\hline Total & 8 & 14 & 30 \\
\hline
\end{tabular}

Tabla 6. Evaluación de los recursos de Química

\begin{tabular}{|l|c|c|c|}
\cline { 2 - 4 } \multicolumn{1}{c|}{} & Primer año & Segundo año & Tercer y cuarto año \\
\hline Excelente & 11 & 29 & 29 \\
\hline Regular & 24 & 9 & 9 \\
\hline Deficiente & 8 & 3 & 3 \\
\hline Total & 43 & 41 & 41 \\
\hline
\end{tabular}

\section{Discusión}

El Ministerio de Educación chileno introduce permanentemente recursos tecnológicos en las aulas del sistema escolar; pese a ello, la incidencia que esto tiene sobre los procesos de enseñanza y aprendizaje, no se reflejan en términos significativos (Ortiz 2017).

Ortega (2011) señala que no podemos plantear que, el objetivo de utilización de las TIC en la materia de Ciencias resida en que el alumno sea capaz de acceder a esas fuentes de información tecnológica. Nuestra labor debe ir más allá y favorecer que el estudiantado sea capaz de seleccionar y gestionar esa información. Desarrollar en el alumno la capacidad de ser buscador, seleccionador y gestor de información relevante para la comprensión de fenómenos físicos y naturales.

Esto plantea un punto de partida que se deja en evidencia, y es el acceso a los recursos tecnológicos, lo cual no se garantiza en los libros de texto analizados porque existe un número importante de recursos no disponibles. Baquero y Gutiérrez (2010) mencionan que el no recibir respuestas sobre alguna acción, desencadena la evitación de la misma; se produce un deterioro en el proceso de aprendizaje. 
En relación a los roles de los recursos tecnológicos se debe reflexionar que, al encontrarnos en la denominada Sociedad de la Información, nos situamos con un mar de información distribuida en diferentes formatos y con elementos multimedia asociados; pero debemos considerar los inconvenientes que estos recursos en línea pueden presentar. Barroso (2004) señala que algunos relacionados con el contenido, no son demasiado estáticos y simplemente consisten en ficheros en formato de texto o pdf; además, si los materiales no se diseñan en forma específica, se puede caer en la potenciación del aprendizaje memorístico; es decir, recursos que asumen un rol meramente informativo.

Esto se observa en los textos analizados, en donde la mayoría de los recursos expuestos poseen un rol informativo, siendo un $78.8 \%$ recursos de Biología y el 83.7\% de Química; dejando una cantidad minoritaria para los roles demostrativos: solo $17.3 \%$ son recursos de Biología y 11.1\% de Química; e interactivos, un 3.8\% recursos de Biología y 5.12\% de Química. Un aporte de los recursos tecnológicos es sacar al estudiante de la abstracción de los contenidos, como Garcés-Ruiz y Martínez (2014) proponen, al mencionar la transformación pedagógica existente gracias a las tecnologías; reconociendo su importancia en el proceso de enseñanza y aprendizaje.

La tecnología nos da la posibilidad de mostrar a los estudiantes tejido celular. Sin necesidad de tener laboratorio, podemos tener software educativo para poder aprender la diferencia entre número atómico y número másico con imágenes y animaciones. Junto a lo anterior, y refrendando a Marc Prensky (2001), los estudiantes procesan la información de forma muy diferente a los adultos.

Pese a que existe relación entre los objetivos de aprendizaje, de los planes y programas, y la mayor parte de los recursos tecnológicos, hay que destacar que existe un importante número de recursos señalados como de calidad regular y deficiente en todos los cursos y en ambas especialidades; siendo un $63.4 \%$ de recursos de Biología y 44.8\% de Química.

Pese a su relevancia como material de apoyo, autores como García-Valcárcel y Hernández (2013) cuestionan a los libros porque ofrecen conocimiento ya elaborado y cerrado; y se le asigna la función de establecer el conocimiento a adquirir, lo que puede limitar las posibilidades más abiertas y constructivas del conocimiento. Así mismo son instrumentos al servicio de los intereses ideológicos y políticos de determinados grupos sociales o del propio Estado; limitando la capacidad crítica y la libertad del profesorado.

Se le puede acusar de ser un material curricular estandarizado, que ordena la vida del aula sin tener en cuenta las características propias del grupo de alumnos, ni del contexto social en que se desarrolla el proceso educativo; y un dispositivo privilegiado de las políticas de control. 


\section{Referencias}

Abarca et al. (2014). Texto del estudiante Biología $3^{\circ}$ y $4^{\circ}$ Medio. Santillana

Baquero, A. y Gutiérrez, G. (2010). Abram Amsel: Teoría de la Frustración y aprendizaje disposicional. Revista Latinoamericana de Psicología, 39(3), 663-667.

Barroso J. (2004). La Red como instrumento de búsqueda de información y de comunicación. En Cabero \& Romero (Coord). Nuevas tecnologías en la práctica educativa (pp. 153). Arial Ediciones.

Cabello, M. (2015). Texto del estudiante Química $1^{\circ}$ Medio. Cal y Canto.

Cabello, M. (2015b). Texto del estudiante Química $3^{\circ}$ y $4^{\circ}$ Medio. Cal y Canto.

Capuano, V. (2011). El uso de las TIC en la enseñanza de las Ciencias Naturales. Facultad de Ciencias Exactas Físicas y Naturales. https://revistas .unc.edu.ar/index.php/vesc/article/ view/335/334

Castells, M. (2001). La era de la información. La Sociedad Red. Vol. 1. Alianza Editorial S.A.

Chaucon, M. y Vargas, R. (2015). Texto del Estudiante Biología $2^{\circ}$ Medio. Santillana.

Estrada, M.; Gómez, H. y Lara, L. (2015). Texto del estudiante Química $1^{\circ}$ Medio. McGraw-Hill.

Garcés-Prettel, M.; Ruiz-Cantillo, R. y Martínez-Avila, D. (2014). Transformación Pedagógica Mediada por Tecnologías de la información y la comunicación. Saber, Ciencia y Libertad, $9(2), 217-227$.

García, A. (1996). Los usos del libro de texto en la práctica docente cotidiana de tercero y cuarto de primaria: Un estudio cualitativo. DIE-CINVESTAV.

García-Valcárcel, A. y Hernández, A. (2013). Recursos tecnológicos para la enseñanza e innovación educativa. Síntesis.

Hernández, R.; Fernández, C. y Baptista, P. (2010). Metodología de la investigación. McGraw Hill.

Instituto de Informática Educativa. Universidad de La Frontera (2006) Una Educación para una Aldea Global. Temuco.

López, F. (2002). El análisis de contenido como método de investigación. XXI. Revista de Educación, (4), 167-180.

López, M. y Pereda S. (2015). Texto del estudiante Biología $1^{\circ}$ Medio. Santillana. 
Meneses, G. (2007). El proceso de enseñanza - aprendizaje: el acto didáctico. Universitat Rovira I Virgili NTIC, interacción y aprendizaje en la universidad. https://www.tdx.cat/bitstream/ handle/10803/8929/Elprocesodeensenanza.pdf? sequence $=32$

Ministerio de Educación (2008). Política de Textos Escolares. Santiago: Chile.

Ministerio de Educación (2019). Bases curriculares de Educación Básica. Santiago: Chile.

Moreira, M. A. (2011). Los efectos del modelo 1: 1 en el cambio educativo en las escuelas. evidencias y desafíos para las políticas iberoamericanas. Revista Ibero-Americana de Educação, (56), 49-74.

Naranjo, G. y Candela, A. (2010). Del libro de texto a las clases de Ciencias Naturales: la construcción de la ciencia en el aula. Papeles de Trabajo sobre Cultura, Educación y Desarrollo Humano, 6 (1), 1-34.

Ortega, J. (2011). Trabajando las Ciencias de la Naturaleza (Biología y Geología) con mapas conceptual. En Marín Díaz, V. (Coord). Cómo trabajar la competencia digital en Educación Secundaria. (pp. 80). Ediciones MAD, S.L.

Ortiz, Y. (2016). Tecnologías de la información y comunicación en estudiantes de Educación Básica de la ciudad de Osorno. Revista Conocimiento Educativo, 3, 55-69. DOI: https:// doi.org/10.5377/ce.v3i0.5645

Ortiz, Y. (2017). Accesibilidad usando las tecnologías de la información y la comunicación. Revista Conocimiento Educativo, 4. DOI: https://doi.org/10.5377/ce.v4i0.5650

Pino, C. y Díaz-Levicoy, D. (2013). Análisis de las actividades propuestas en dos textos escolares de primer año medio para la enseñanza de la célula. Revista Electrónica Diálogos Educativos, 13 (26), 18-30.

Pino, C.; Díaz-Levicoy, D. y Piñeiro, J. L. (2014). Los gráficos estadísticos como articuladores del currículo escolar. Revista chilena de Educación Científica, 13 (2), 9-18.

Prensky, M. (2001). Nativos digitales, inmigrantes digitales. On the horizon, 9 (5), 1-7.

Ramirez, A. (2011). Las TIC y el Desarrollo de Competencias Básicas en Educación Secundaria. En Marín Díaz V. (Coord) Cómo trabajar la competencia digital en Educación Secundaria. (pp.17-20). Ediciones MAD, S.L.

Waldegg-Casanova, G. (2002). El uso de las nuevas tecnologías para la enseñanza y el aprendizaje de las ciencias. Revista electrónica de investigación educativa, 4 (1), 01-22. http:// www.scielo.org.mx/scielo.php?script=sci_arttext\&pid=S1607-40412002000100006 